Annales Geophysicae (2001) 19: 11-15 C European Geophysical Society 2001

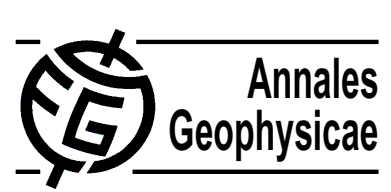

\title{
Observation of isotropic electron temperature in the turbulent $E$ region
}

\author{
S. Saito, S. C. Buchert, S. Nozawa, and R. Fujii \\ Solar-Terrestrial Environment Laboratory, Nagoya University, Furo-cho, Chikusa-ku, Nagoya, 464-8601, Japan
}

Received: 21 January 2000 - Revised: 26 October 2000 - Accepted: 20 November 2000

\begin{abstract}
Using EISCAT radar data, we find that electrons are strongly heated in the magnetic field-line direction during high electric field events. The remote site data show that the electron temperature increases in almost the same way in the field-perpendicular direction; electron heating by $\mathrm{E}$ region plasma turbulence is isotropic. We discuss the implications of our observation for the "plasmon"-electron as well as the wave Joule heating models of the anomalous electron heating in the E region.
\end{abstract}

Key words. Ionosphere (auroral ionosphere; plasma temperature and density; plasma waves and instabilites)

\section{Introduction}

The knowledge and understanding of irregularities in the $\mathrm{E}$ region was recently reviewed (Sahr and Fejer, 1996). Usually dominant is the modified two-stream (or Farley-Buneman) instability (MTSI). This causes echoes in the HF and VHF ranges at angles nearly perpendicular to the geomagnetic field. Coherent radars like the STARE and also SuperDARN detect such echoes and measure geophysical parameters such as the electric field. Understanding of the non-linear plasma physics involved in the MTSI is not only essential for the correct analysis of these radar data, the unstable $\mathrm{E}$ region is also an important natural example of plasma turbulence.

The plasma in the E region is strongly influenced by collisions between charged and neutral particles. This normally enforces thermal equilibrium below about $110 \mathrm{~km}$, i.e., temperatures of neutrals, ions, and electrons, $T_{n}, T_{i}$, and $T_{e}$, respectively, are equal. Only at higher altitudes can selective heat sources, like frictional heating of the ions or ionizing solar radiation, raise $T_{i}$ or $T_{e}$ above $T_{n}$. Therefore, strong enhancements of $T_{e}$ below $110 \mathrm{~km}$, first reported by Schlegel and St.-Maurice (1981) using the Chatanika radar, were unexpected. Further observations were made with the EISCAT radar (Igarashi and Schlegel, 1987; Williams et al., 1990;

Correspondence to: S. Saito (susaito@stelab.nagoya-u.ac.jp)
Jones et al., 1991). Davies and Robinson (1997) also showed the electron heating in the $\mathrm{E}$ region on a statistical basis using 900 hours of EISCAT CP-1 and CP-2 data. Generally, it was found that $T_{e}$ starts to increase when the electric field strength $|\boldsymbol{E}|$ exceeds the threshold for excitation of the MTSI, $E_{t h} . T_{e}$ increases approximately linearly with $|\boldsymbol{E}|-E_{t h}$.

St.-Maurice et al. (1981) first realized that the electron heating is due to electrostatic waves generated in the MTSI. From in-situ observations with rockets, Primdahl and Bahnsen (1985) argued that the heating can be explained by assuming an anomalous collision frequency $v^{\star}$ for the electrons drifting in the background electric field. $v^{\star}$ parameterizes all macroscopic effects due to waves. Also, Robinson (1986) put forward a more self-consistent model where all macroscopic effects can be parameterized by a $v^{\star}$ representing collisions between electrons and "plasmons". The model is supported by mean-field turbulence theory (Sudan, 1983) and was further extended (Robinson and Honary, 1990). Ogawa et al. (1980) has shown that when the electric field is strong, the effective collision frequency of electrons should be enhanced by a factor of 6 to explain the observed aspect angle dependence of phase velocities of $\mathrm{E}$ region irregularities. Because the wave fronts seen at VHF frequencies are close to field-aligned, Farley (1985) has argued that the $v^{\star}$ applies only to the field-perpendicular direction. The E region turbulent transport coefficient should be strongly anisotropic.

St.-Maurice (1987) noted that extraordinary heating results from normal electron-neutral collisions when the electrons are driven by the background electric field $\boldsymbol{E}_{0}$ as well as by wave fields $\delta \boldsymbol{E}$. For wave frequencies much less than the electron gyrofrequency (as is the case for VHF and HF waves), the electron motion would not become ther$\mathrm{mal} /$ random unless the electrons are scattered in collisions with neutrals. This viewpoints contrasts to some extent with a Kolmogoroff-type approach, where waves with random phases ("plasmons") thermalize electrons and cause the heating directly, (even if there are no electron-neutral collisions).

The in-situ observed VHF wave amplitudes and density 
fluctuations are often too weak to explain the heating rates derived from radar observations if the electrons are driven in the perpendicular direction only. St.-Maurice and Laher (1985) postulated therefore the existence of a small wave field component parallel to the magnetic field, $\delta \boldsymbol{E}_{\|}$. This would greatly enhance the average Joule heating rate

$$
\begin{aligned}
W_{e} & =\left\langle\delta \boldsymbol{j}_{\perp} \cdot \delta \boldsymbol{E}_{\perp}\right\rangle+\left\langle\delta \boldsymbol{j}_{\|} \cdot \delta \boldsymbol{E}_{\|}\right\rangle \\
& =\sigma_{P}\left\langle\delta E_{\perp}^{2}\right\rangle+\sigma_{\|}\left\langle\delta E_{\|}^{2}\right\rangle
\end{aligned}
$$

because the parallel conductivity $\sigma_{\|}$is much bigger than the Pedersen conductivity $\sigma_{P}, \sigma_{\|} \gg \sigma_{P}$. The existence of $\delta \boldsymbol{E}_{\|}$ was noted as an open question in the text book by Kelley (1989).

Experimental studies of anisotropy have, so far, concentrated on the waves by using radar backscatter from the ground. VHF radar echoes are observed at angles further away from the perpendicular to the earth's magnetic field than expected from linear theory. Also, at higher frequencies, $930 \mathrm{MHz}$, the backscattering is not only surprisingly strong but occurs at aspect angles even further away from the perpendicular (Moorcroft and Schlegel, 1988; Eglitis et al., 1996). Obviously the E region plasma turbulence becomes more isotropic at shorter wavelengths.

In this work we search for a directional dependence of the electron temperature during heating events using tristatic EISCAT UHF radar. If we find anisotropic temperatures, the heating would have to be caused by perpendicular wave fields directly because electron-neutral collisions heat isotropically irrespective of the direction of $\boldsymbol{E}_{0}$ and $\delta \boldsymbol{E}$. On the other hand, the finding of isotropic temperatures would be consistent with St.-Maurice's model as well as with Robinson's electron-plasmon scattering. However, for the latter viewpoint, a finding of isotropic temperatures implies restrictions which will be discussed later in this work. Thus our analysis can potentially help to decide between two hitherto indistinguishable interpretations of experimental results.

In the next section, we present a comparison of electron temperatures measured with different aspect angles to the geomagnetic field, $\boldsymbol{B}$. In Sect. 3 we interpret the results of Sect. 2 and discuss the implications for the E region plasma turbulence.

\section{Observations}

Figure 1 shows a summary of the data obtained by the EISCAT CP-1-I experiment from 1200 to 1700 UT on June 12, 1990 ,when we found several distinctive $T_{e}$ enhancement events. The antenna of the transmitting and receiving site in Troms $\varnothing$, Norway, was directed along $\boldsymbol{B}$. The remote antennas in Kiruna, Sweden and Sodankylä, Finland, measured alternately a common volume at $278 \mathrm{~km}$ altitude for electric field determination and six common volumes in the E region that can be used to detect any plasma anisotropy. The dwell times of the remote antennas are 50 to $60 \mathrm{~s}$. For E region measurements, the Troms $\emptyset$ site uses a five-pulse code with a range resolution of approximately $3 \mathrm{~km}$. The remote sites receive a long pulse of $350 \mu \mathrm{s}$. The height resolution of the remote data is determined by the antenna beam width which is $0.8^{\circ}$. In the $\mathrm{E}$ region, this corresponds to approximately $2.9 \mathrm{~km}$ for the Kiruna antenna and $5.5 \mathrm{~km}$ for Sodankylä. Thus the height resolutions of both Troms $\varnothing$ and remote data are comparable.

During the period shown in Fig. 1, the electric fields measured in the F region are generally high, until 1555 UT. Strong electron heating between 100 and $115 \mathrm{~km}$, with amplifications at about 1240, 1450, and 1515 UT, are associated with high $|\boldsymbol{E}|$. In the most intense event, $T_{e}$ reaches about $1700 \mathrm{~K}$ between 1454 and 1455 UT. Ions are heated up by frictional (Joule) heating above $115 \mathrm{~km}$. The electron temperatures in the lower E region below about $115 \mathrm{~km}$ are generally enhanced over both the neutral and ion background temperatures.

Using the E region measurements from the remote EISCAT sites, we have checked the thermal isotropy of the electrons and also the ions. Figures 2 and 3 show scatter plots of temperatures obtained simultaneously from two different sites using all the data from 0800 UT, June 12 to 1345 UT, June 13, 1990. At $108 \mathrm{~km}$, the aspect angle of the Sodankylä data is $39^{\circ}$ while that of Troms $\emptyset$ is $0^{\circ}$. In Fig. 2 (left panel) the electron temperatures from these two directions are compared with each other. At the highest $T_{e}$ of $1700 \mathrm{~K}$, observed by Troms $\varnothing$, no simultaneous observation by the remote sites is available at this altitude; therefore the plotted $T_{e}$ reaches only about $1100 \mathrm{~K}$. In Fig. 2 (right panel) the ion temperatures from the two sites are compared. The comparisons of the electron and the ion temperatures at $108 \mathrm{~km}$, between Troms $\emptyset$ and Kiruna where the aspect angle is $35^{\circ}$, are shown in Fig. 3 (left and right), respectively. Occasionally, some preferentially transverse ion heating seems to occur even at this low altitude but to a much lesser degree than the electron heating. The important point in this context is that the plots indicate clearly the isotropy of $T_{e}$ at all temperatures. Although not shown here, comparisons at other altitudes are similar.

\section{Discussion}

The $\mathrm{E}$ region plasma waves, which are assumed to be the cause of electron heating, are highly anisotropic. From coherent radar observations it is known that at wavelengths above about a meter the back-scattered wave amplitudes fall off within aspect angles of about $2^{\circ}$ away from the magnetic field. From this, one might expect anisotropic electron temperatures. Nevertheless, our results show that the $T_{e}$ enhancement is clearly isotropic.

If electrostatic waves heat electrons directly, the wavelengths must be smaller than the electron gyroradius $r_{g}$ $(\sim 1 \mathrm{~cm})$. Otherwise the wave-particle interaction is inefficient in giving electrons enough energy to heat them up. For long wave lengths $\left(\gg r_{g}\right)$ and low frequencies $(\ll$ the electron gyrofrequency $\Omega_{c e} \sim 9 \mathrm{MHz}$ ) the electrons perform 


\section{EISCAT CP1-I on 12/06/1990}
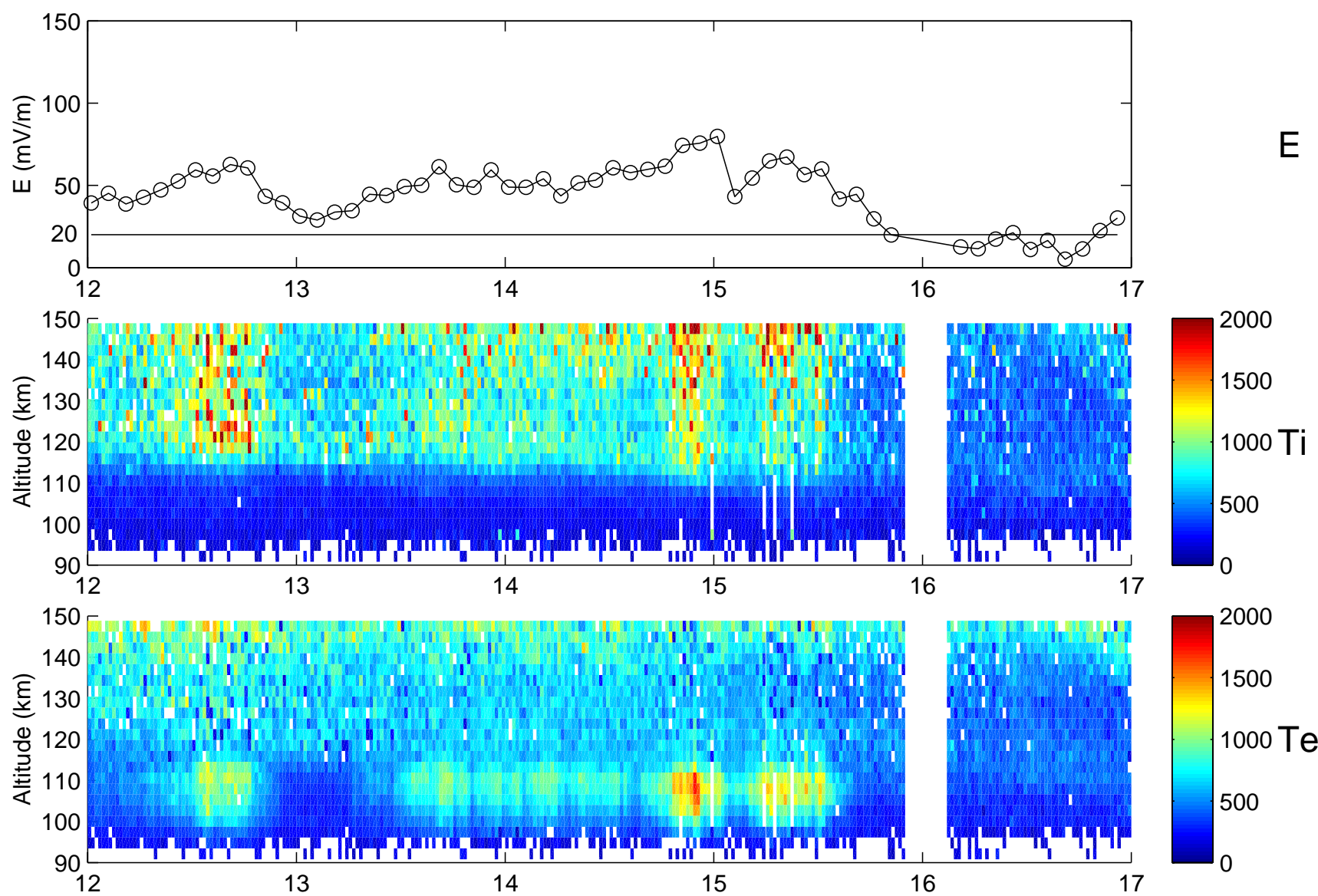

Fig. 1. Overview of a data set with long periods of elevated $T_{e}$ in the lower E region. The top panel shows $|\boldsymbol{E}|$ with a time resolution of 5 minutes. The approximate threshold of the MTSI is about $20 \mathrm{mVm}^{-1}$. The middle and bottom panels show the electron and ion temperatures, respectively.

alternating, deterministic $\boldsymbol{E} \times \boldsymbol{B}$ drifts. In-situ observations show that the wave amplitudes at shorter wavelengths decrease substantially (Pfaff et al., 1984). Extrapolating this trend to (unobserved) $\mathrm{cm}$-wavelengths, we find that direct heating by such waves is an unlikely explanation for our observed isotropic enhancements of $T_{e}$.

If we also take into account electron-neutral collisions, the strong but slow and anisotropic Farley-Buneman waves can lead to isotropic electron heating. The electrons' deterministic $(\boldsymbol{E}+\delta \boldsymbol{E}) \times \boldsymbol{B}$ drift gets in each elastic collision with a neutral completely and isotropically randomized. Due to the huge mass difference between electrons and neutrals, an electron loses practically no energy in elastic collisions but the energy is converted from flow energy to heat. About every 100th collision with a neutral is inelastic, exciting vibrational and rotational modes of $\mathrm{N}_{2}$ and $\mathrm{O}_{2}$ molecules as well as fine structure levels of $\mathrm{O}$ (Schunk and Nagy, 1978). In an inelastic collision, the electron transfers typically a large fraction of its energy to the neutrals. The efficiency of this process ensures that $T_{e}$ is hardly elevated above neutral temperature in the lower E region even when there are DC elec- tric fields of the order of $100 \mathrm{mV} / \mathrm{m}$ (which is typical for the auroral zone). The amplitudes of Farley-Buneman waves $\delta E$ do not become larger than the DC background field even in the non-linear stage of the instability. Therefore, purely perpendicular waves cannot explain the large observed enhancements of $T_{e}$. Consequently, St.-Maurice (1987) postulated a field-parallel component of $\delta \boldsymbol{E}, \delta \boldsymbol{E}_{\|}$, which would heat electrons very efficiently due to high parallel transportability as discussed in the introduction. Our observation of isotropic $T_{e}$ enhancements is in accordance with St.-Maurice's model, although we cannot add any quantitative estimates of the size of $\delta \boldsymbol{E}_{\|}$.

\section{Summary and Conclusion}

We have shown that the electron temperatures observed in the $\mathrm{E}$ region are clearly isotropic over aspect angles from $0^{\circ}$ to about $40^{\circ}$ even when they are strongly enhanced. If the actual heating process is through electron-neutral collisions which have no directional preferences, it is natural to expect isotropic $T_{e}$. The very high heating rates that we 

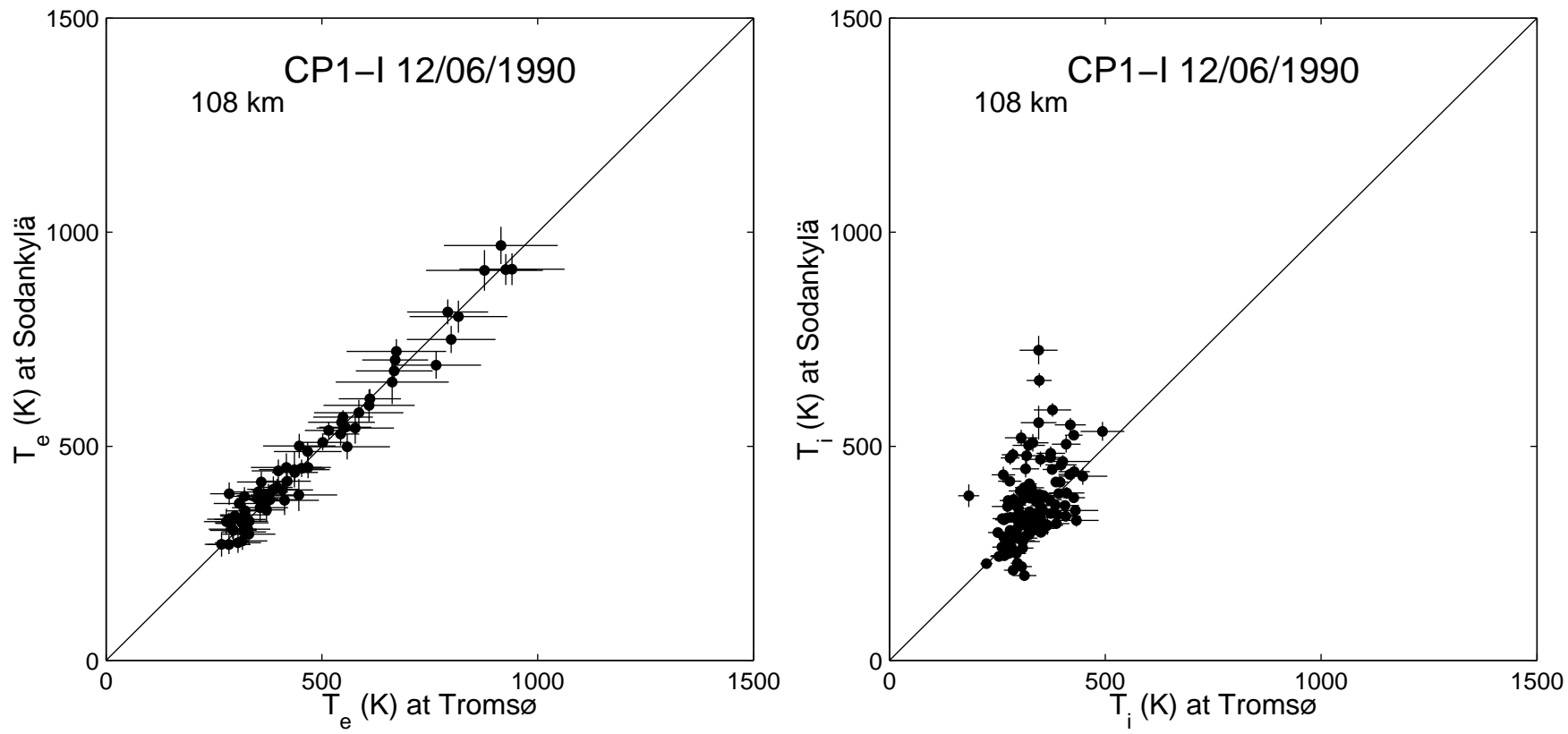

Fig. 2. Left panel: Scatter plot of the electron temperatures from different aspect angles observed with the Troms $\varnothing$ and Sodankylä EISCAT sites. The errors in fitting to the IS spectra are also plotted. Right panel: The same as the left panel, but the ion temperatures are compared.
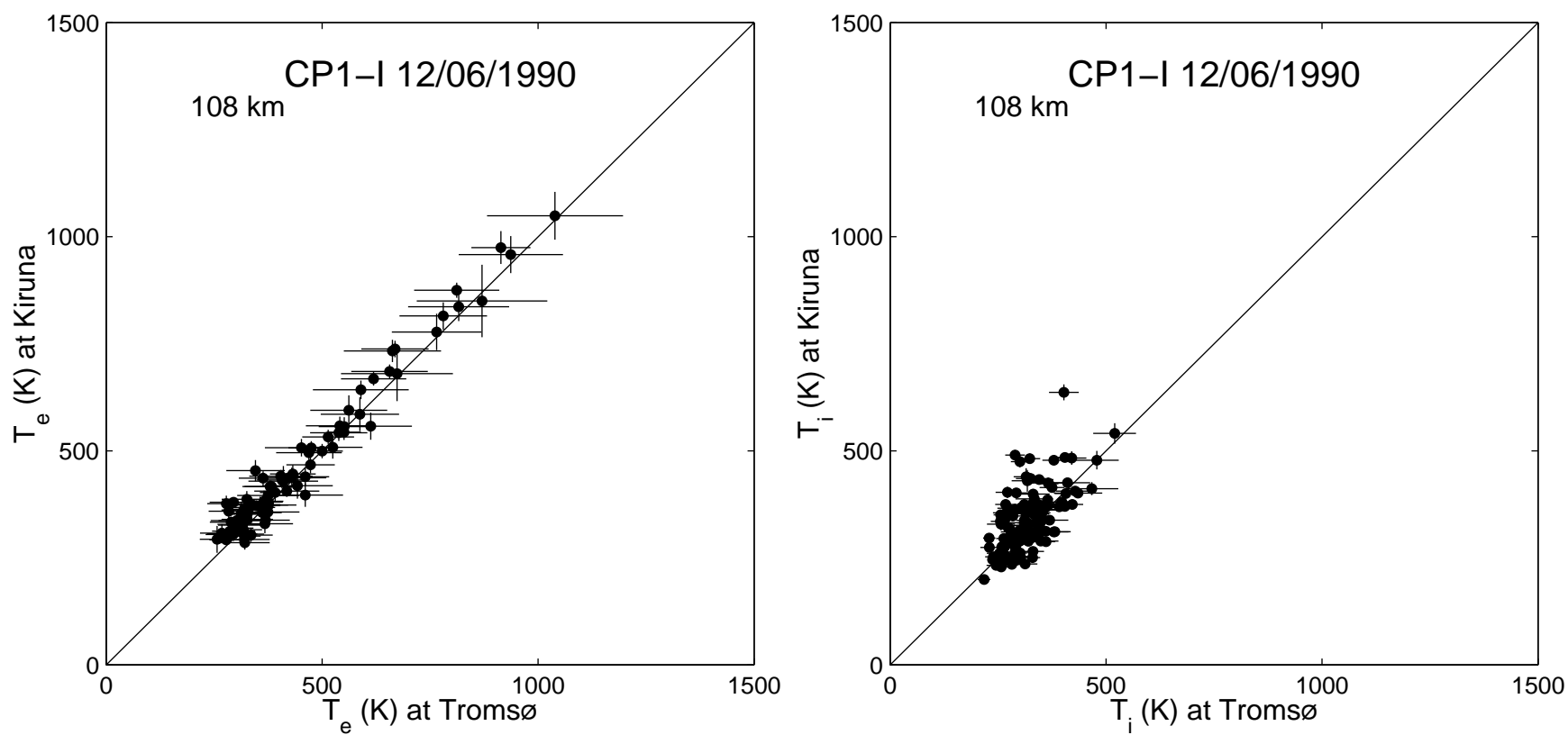

Fig. 3. The same as Fig. 2, but Troms $\varnothing$ and Kiruna data are compared.

derive from the observed $T_{e}$, using the standard model for isotropic temperatures (Schunk and Nagy, 1978), suggests that Farley-Buneman waves have an electric field component both perpendicular and parallel to $\boldsymbol{B}$ as proposed by St.Maurice (1987). If, on the other hand, $T_{e}$ enhancement is due to a direct scattering of electrons by wave fields not involving electron-neutral collisions, our observation implies that these waves should be isotropic.

We think that our result of isotropic $T_{e}$ is remarkable in light of the known strong anisotropy of the Farley-Buneman waves, the cause of heating. We feel that further clarifications of the underlying heating processes are needed in the future.

Acknowledgement. We are indebted to the director and staff of EISCAT for operating the facility and supplying the data. EISCAT is an international association supported by Finland (SA), France (CNRS), the Federal Republic of Germany (MPG), Japan (NIPR), Norway (NFR), Sweden (NFR), and the United Kingdom (PPARC). This study was financially supported by the Grant-in-Aid for Scientific Research A (12373002), B (11440144), and C (11640411) by 
the Ministry of Education, Science, Sports and Culture, Japan. Topical Editor Mark Lester thanks two referees for their help in evaluating this paper.

\section{References}

Davies, J. A. and Ronbinson, T. R., Heating of the high-latitude ionospheric plasma by electric fields, Adv. Space Res. 20, 11251128, 1997.

Eglitis, P., McCrea, W., Robinson, T. R., Jones, T. B., Schlegel, K., and Nygren, T., Flow dependence of COSCAT spectral characteristics, J. Atmos. Terr. Phys. 58, 189-203, 1996.

Farley, D. T., Theory of equatorial electrojet plasma waves: new developments and current status, J. Atmos. Terr. Phys. 47, 729744, 1985.

Igarashi, K. and Schlegel, K., Electron temperature enhancements in the polar E region measured with EISCAT, J. Atmos. Terr. Phys. 49, 273-280, 1987.

Jones, B., Williams, P. J. S., Schlegel, K., Robinson, T., and Häggström, I., Interpretation of enhanced electron temperatures measured in the auroral E region during the ERRRIS campaign, Ann. Geopys. 9, 55-59, 1991.

Kelley, M. C., The Earth's ionosphere, Academic Press, 1989.

Moorcroft, D. R. and Schlegel, K., E region coherent backscatter at short wavelength and large aspect angle, J. Geophys. Res. 93, 2005-2010, 1988.

Ogawa, T., Balsley, B. B., Ecklund, W. L., Carter, D. A., and Johnston, P. E., The effects of the resonance broadening of FarleyBuneman waves on electron dynamics and heating in the auroral E-region, Geophys. Res. Lett. 7, 1081-1084, 1980.

Pfaff, R. F., Kelley, M. C., Fejer, B. G., Kudeki, E., Carlson, C. W., Pedersen, A., and Häusler, B., Electric field and plasma density measurements in the auroral electrojet, J. Geophys. Res. 89, 236244, 1984.
Primdahl, F. and Bahnsen, A., Auroral E region diagnosis by means of nonlinearly stabilized plasma waves, Ann. Geopys. 3, 57-62, 1985.

Robinson, T. R., Toward a self-consistent non-linear theory of radar auroral backscatter, J. Atmos. Terr. Phys. 48, 417-422, 1986.

Robinson, T. R. and Honary, F., A resonance broadening kinetic theory of the modified two-stream instability: Implications for radar auroral backscatter experiments, J. Geophys. Res. 95, 10731085, 1990.

Sahr, J. D. and Fejer, B. G., Auroral electrojet plasma irregularity theory and experiment: A critical review of present understanding and future directions, J. Geophys. Res. 101, 26893-26909, 1996.

Schlegel, K. and St.-Maurice, J.-P., Anomalous heating of the polar E region by unstable plasma waves, 1 . Observations, J. Geophys. Res. 86, 1447-1452, 1981.

Schunk, R. W. and Nagy, A. F., Electron temperature in the F region of the ionosphere: Theory and observations, Rev. Geophys. and Space Phys. 16, 355-399, 1978.

St.-Maurice, J. P., A unified theory of anomalous resistivity and Joule heating effects in the presence of E-region irregularities, J. Geophys. Res. 92, 323-327, 1987.

St.-Maurice, J.-P. and Laher, R., Are observed broadband wave amplitudes large enough to explain the enhanced electron temperatures of the high-latitude E region?, J. Geophys. Res. 90, $2843-$ 2850, 1985.

St.-Maurice, J. -P., Schlegel, K., and Banks, P. M., Anomalous heating of the polar E region by unstable plasma waves, 2. Theory, J. Geophys. Res. 86, 1453-1462, 1981.

Sudan, R. N., Unified theory of type I and type II irregularities in the equatorial electrojet, J. Geophys. Res. 88, 4835-4860, 1983.

Williams, P. J. S., Jones, G. O. L., Jones, B., and Opgenoorth, H., High-resolution measurements of magnetospheric electric fields, J. Atmos. Terr. Phys. 52, 439-448, 1990. 